\title{
Book review: Hills, H. (2001) Team-Based Learning
}

\author{
Susan Jones
}

In the book Team-Based Learning, Hills looks at what makes a team successful, and how teams can improve their performance. His focus in the book is two-fold: not just the components of a successful team, but more importantly, how teams (and individuals in those teams) can learn and improve their performance. The book is generally quite easy to read, and the author definitely has a fondness for catchy metaphors, mnemonics, stories, etc. While the concepts are not necessarily new, he has his own terminology and style and presents the ideas using straightforward language rather than embedded in a lot of theory.

Hill's background is very much in the corporate world, and much of the book draws on his experiences in this area, but many of the topics he covers such as stages of team formation, various roles in a team, role of the leader, etc, are concepts that apply to every type of team work, including at the university.

According to Hill, teams are a central component of today's world. Every organization, whether private or public, uses teams to get things done. Teams that learn provide fulfillment and satisfaction for all the members, and therefore lead to growth and development for the individual as well as the organization.

He emphasizes the point that we need to turn 'good' teams into teams that improve. Teams that are good from the start may not really learn much or may rely too heavily on one person, neither of which is the desired outcome of teamwork. Teams that improve actually learn something in the process. One major outcome of any collaborative experience ought to be to ensure that each individual team member can learn and gain satisfaction from the experience.

The book is divided into 3 parts:

Part One - The Foundation for Team-Based Learning

Part Two - The Building Blocks and Tools for Team-Based Learning

Part Three - The Roles of Those Involved in Team-Based Learning

From the names of the three sections, it is easy to see that Hill's focus is as much on the learning aspect of teamwork as on the successful achievement of goals.

Each chapter begins with an overview and ends with a summary of the main ideas of the chapter. If you only want to read one or two of the chapters, or if you want to quickly find out what a chapter will cover, this can be a useful way to find the information that you want or skip those chapters that are not of interest. It does tend to feel a little redundant if you are reading the whole book, but on the positive side, it provides a succinct overview of each chapter.

Chapter one is an introduction to the concept of team and how historically teamwork has been an important contributor to individual development and learning. Chapters two, three and four look at some of the building blocks of team learning; how teams are formed and the roles that exist in any team (based on Belbin ${ }^{1}$ ); the effect of different personality types on the team learning process, and how the culture of the team or the workplace or the organization can affect the behavior of the team members.

One very interesting point that Hill makes in the book is to note that a team made up of high-flyers is not necessarily going to be the best team. In fact, it could have some of the biggest problems because everyone on the team in competing or trying to be in charge, and as a result, no one is happy and no one is learning. Likewise, teams that rely on only one or two key people can often find themselves in a bind if something happens to that person. On the other hand, a team made up of people all filling different roles and all learning from each other and sharing tasks, will be able to accomplish much more than any of them could have done individually. 
The ultimate goal of any team ought to be personal fulfillment and learning for every individual team member, not just finishing the task. We have to recognize what kind of roles we like and what kind of personality we have, but we also have to be willing to work with others and be open to other ways of doing things and be able to adapt our own style to allow the greatest achievement for everyone in the team.

In chapter five, Hill defines team-based learning, distinguishes it from individual learning and explains some of the benefits that can be achieved when it is successful. Here he introduces another of his mnemonics for a model for team interaction - TRUST (trust, respect, understanding, spirit, teams).

Chapters six and seven cover effective learning and how different learning theories can contribute to successful team learning, and Hill identifies some of the barriers to successful team learning such as language, misunderstandings and emotional issues.

The last section of the book looks more closely at the roles of the team leader, the individual and the training department. Chapter eight looks at the various roles that the team leader must play, creating the team culture, and tasks that must be managed. In chapter nine, the roles of individuals, both in contributing to the team process and motivating other members of the team, are outlined. The final chapter, looking at the role of the training department, is once again metaphor laden and squarely situated in the workplace.

1Belbin, M. (1981). Management Teams, Why They Succeed or Fail, London: Heinemann. 